\title{
Bilateral Pulmonary Hydatid Cyst in a Young Child: A Rare Case Report from North India
}

\author{
Priya Datta ${ }^{1}$ Bhawna Sharma ${ }^{1}$ Nitin James Peters ${ }^{2}$ Sumeeta Khurana ${ }^{10}$ Rakesh Sehgal ${ }^{1}$ \\ 1 Department of Medical Parasitology, Postgraduate Institute of \\ Medical Education and Research, Chandigarh, India \\ 2 Department of Pediatric Surgery, Postgraduate Institute of Medical \\ Education and Research, Chandigarh, India

\begin{abstract}
Address for correspondence Bhawna Sharma, MBBS, MD, Department of Medical Parasitology, Postgraduate Institute of Medical Education and Research, Chandigarh, 160012, India (e-mail: 34bhawnasharma@gmail.com).
\end{abstract}

J Lab Physicians 2022;14:348-350.
Abstract
Keywords
- echinococcosis
- hydatid cyst
- bilateral
- pulmonary
- serology

Echinococcosis or hydatid disease is caused by the larval stage of the dog tapeworm, that is, Echinococcus granulosus, E. multilocularis, E. vogeli, or E. oligarthrus. Echinococcus granulosus causes cystic echinococcosis, which has a worldwide distribution. Liver is the most common site, affecting approximately two-third of the patients, whereas lung involvement is seen in approximately $25 \%$ of cases. This case is a very rare scenario of bilateral pulmonary hydatid cysts in a young child having exposure to a pet dog with negative hydatid serology and normal eosinophil count.

\section{Introduction}

Echinococcosis or hydatid disease is caused by larval stage of the dog tapeworm, that is, Echinococcus granulosus, E. multilocularis, E. vogeli, or E. oligarthrus. Echinococcus granulosus causes cystic echinococcosis (CE), which has a worldwide distribution. The seropositivity for echinococcosis increased from 15 to $28.6 \%$ over 20 -year period in North India. Though hydatid cysts can involve any site of the body, liver is the most common site affecting approximately two-third of the patients, whereas lung involvement is seen in approximately $25 \%$ of cases. Most pulmonary cysts are located in the lower lobes (posterior $>$ anterior) and it is unilateral in $80 \%$ of pulmonary echinococcosis. Pulmonary symptoms are nonspecific and the diagnosis is made by correlating epidemiological history, clinical manifestations, imaging tests, and serological tests. In the present case report, we report a young child presenting with bilateral pulmonary hydatid cyst having negative hydatid serology with normal eosinophil counts.

published online February 9, 2022
DOI https://doi.org/ $10.1055 / \mathrm{s}-0042-1742420$. ISSN $0974-2727$.

\section{Case Report}

A 7-year-old boy, resident of Ladakh, North India, presented to pediatric emergency with complaints of pleuritic chest pain for the last 1 month. This pain was localized on the left side of chest, throbbing and penetrating in nature. There was no history of associated fever, cough, breathlessness, abdominal pain, vomiting, or loose stools. The child's parents informed about the presence of a pet dog at home. At the local hospital, chest X-ray showed bilateral paramediastinal well-defined soft tissue opacities, up to middle lung field on the right side and peripheral lung field on the left side. The child was referred to the Postgraduate Institute of Medical Education and Research (PGIMER), Chandigarh. X-ray was repeated at PGIMER which showed similar findings (- Fig. 1). On contrast-enhanced computed tomography, bilateral wellcapsulated symmetrical cystic mass, showing fat and fluid density was seen arising from the posterior mediastinum. A provisional diagnosis of bilateral pulmonary echinococcosis was made. Serology (immunoglobulin G [IgG] antibodies (c) 2022. The Indian Association of Laboratory Physicians. All rights reserved.

This is an open access article published by Thieme under the terms of the Creative Commons Attribution-NonDerivative-NonCommercial-License, permitting copying and reproduction so long as the original work is given appropriate credit. Contents may not be used for commercial purposes, or adapted, remixed, transformed or built upon. (https://creativecommons.org/ licenses/by-nc-nd/4.0/)

Thieme Medical and Scientific Publishers Pvt. Ltd., A-12, 2nd Floor, Sector 2, Noida-201301 UP, India 


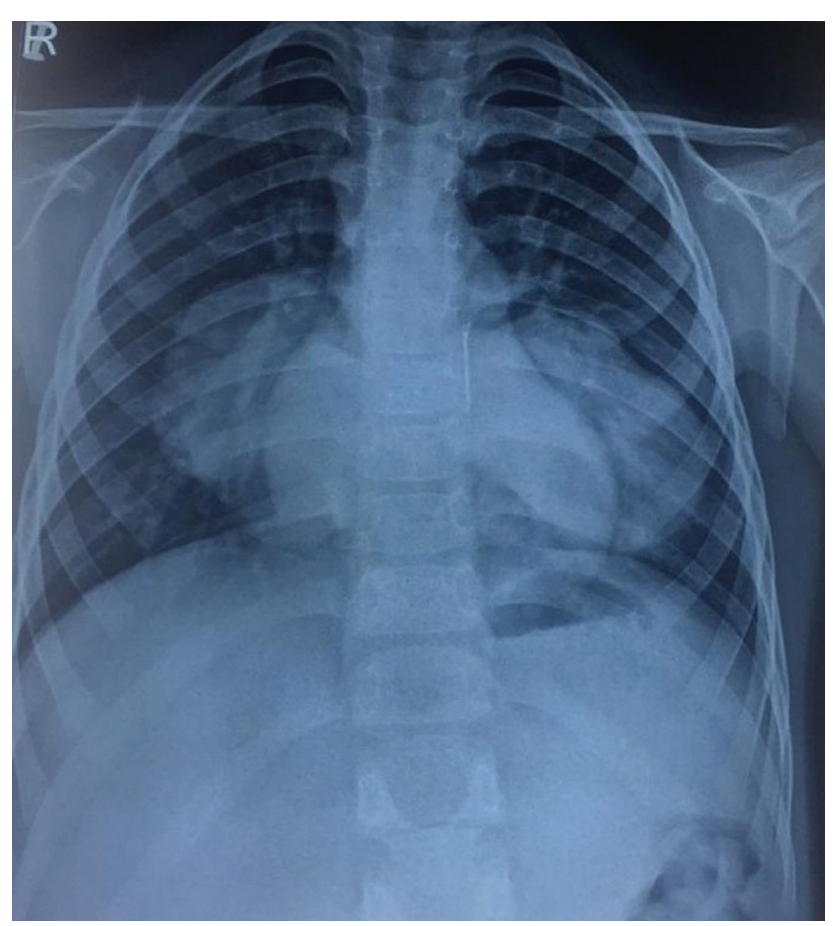

Fig. 1 Chest X-ray posteroanterior view showing bilateral hydatid cysts.

against hydatid) was done on the patient's serum which showed negative results (Ridascreen Echinococcus IgG ELISA kit). Other blood investigations were all within the normal range with $1.9 \%$ eosinophils. Ultrasound of the abdomen was normal. Pediatric surgeon's opinion was taken and the patient was advised bilateral thoracotomy along with partial cystectomy and marsupialization. On right posterolateral thoracotomy, $5 \times 4 \mathrm{~cm}$ cyst was identified in the lower lobe. The cyst cavity was opened and hydatid membranes were evacuated. On left posterolateral thoracotomy, a $6 \times 5 \mathrm{~cm}$ cyst was visualized in the lower lobe and the same procedure as that of right side was followed. Bilateral intercostal drainage tubes were put. A part of both the cysts along with fluid was sent to Department of Medical Parasitology, PGIMER. On wet mount of cystic fluid, plenty of scolices and hooklets were seen, resembling E. granulosus (-Fig. 2). Histopathological examination of right and left pericyst showed lamellated acellular eosinophilic membrane with the presence of germinal layer, features suggestive of hydatid cyst. Postsurgery, the patient was given albendazole $150 \mathrm{mg}$ BD for 3 weeks then stopped for 1 week and again started for 3 weeks, injection augmentin $450 \mathrm{mg}$ three times a day and injection amikacin $22.5 \mathrm{mg}$ intravenous once daily for 7 days were also given. The patient was discharged after 1 week under stable conditions.

\section{Discussion}

Hydatid disease is endemic mainly in the Mediterranean countries (particularly Greece), the Middle East, the Baltic areas, South America, India, northern China, and other sheep-raising areas. However, owing to increased travel and tourism all over the world, it can be found anywhere,

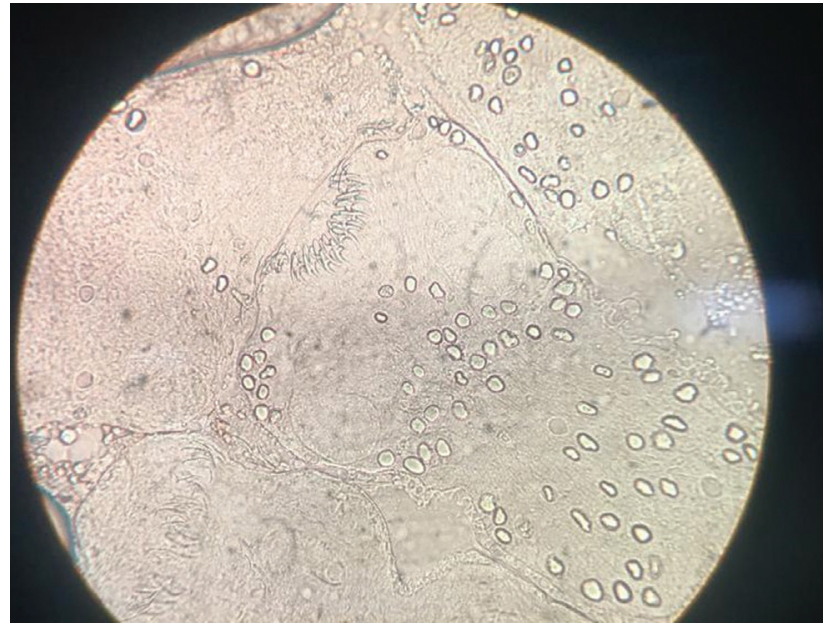

Fig. 2 Wet mount of cystic fluid from right pulmonary cyst showing hooklets of Echinococcus granulosus (under 40x magnification).

even in developed countries. ${ }^{1}$ In India, the highest prevalence of hydatid disease was reported from Andhra Pradesh and Tamil Nadu. ${ }^{2}$ In case of pulmonary hydatid cysts, solitary cysts are more common than multiple cysts and also unilateral cysts occur more frequently than bilateral. ${ }^{3}$ In $20 \%$ of cases, it can be bilateral and multiple cysts in $30 \%$ of cases. ${ }^{4}$ There are very few studies available regarding the prevalence of pulmonary hydatidosis from India. Punia et al did one study from North India from 2003 to 2012 (10 years), in which only eight cases of pulmonary hydatid cyst were reported and two of them had bilateral involvement. ${ }^{5}$ Similarly, a retrospective study (2004-2015) was done from our institute only, which showed that out of all cases of CE, pulmonary involvement was seen in only $8.7 \%$ of cases. ${ }^{6}$ In an Indian study done by Rao et al, out of total 117 patients of hydatid disease from central India, lung involvement was seen in 17 patients (14.53\%) either solitarily or in association with other organs. ${ }^{7}$ In our case, there are bilateral pulmonary cysts without hepatic involvement which is a very rare scenario. Unlike an adult, lung involvement is more common than liver in children, with frequencies of 64 and $28 \%$, respectively. ${ }^{8}$ Radiology and serology are the principal diagnostic modality used to confirm the diagnosis. Typical chest $\mathrm{X}$-ray findings of uncomplicated pulmonary hydatid disease are single or multiple homogenous round mass with smooth borders as seen in our case also. Routine blood tests are usually nonspecific and only $15 \%$ of patients show eosinophilia due to leakage of antigenic material. ${ }^{9}$ Serological tests include enzyme-linked immunosorbent assay, latex agglutination, indirect hemagglutination test, and an immunoblot assay using lentil-lectin purified glycol proteins. These serological methods have a higher sensitivity (85-98\%) for hepatic hydatid disease, but lower (50-60\%) for the pulmonary cases. In our case, serology came out to be negative. Surgical removal of cyst and its fluid examination can establish the diagnosis of hydatid disease by demonstrating the protoscolices, hooklets, or cyst membranes as in our case. This is a very rare case of bilateral multiple pulmonary hydatid cysts in a young child having exposure to a dog living in sheep 
rearing area, with normal eosinophil count and negative hydatid serology. The given case highlights that in such areas, though bilateral hydatid cysts are rare, it should be considered in the differential diagnosis of such lesions on chest $\mathrm{X}$ rays. Furthermore, one should not rely on serology for confirmation of diagnosis as it could be negative in these cases.

\section{Funding}

None.

\section{Conflict of Interest}

None declared.

\section{References}

1 Huizinga WKJ, Grant CS, Daar AS. “Hydatid disease.". In: Morris PJ, Wood WC, eds. Oxford Textbook of Surgery. 2nd ed. Oxford, UK: Oxford University Press;2000:3298-3305
2 Amir-Jahed AK, Fardin R, Farzad A, Bakshandeh K. Clinical echinococcosis. Ann Surg 1975;182(05):541-546

3 Erdem CZ, Erdem LO. Radiological characteristics of pulmonary hydatid disease in children: less common radiological appearances. Eur J Radiol 2003;45(02):123-128

4 Garg MK, Sharma M, Gulati A, et al. Imaging in pulmonary hydatid cysts. World J Radiol 2016;8(06):581-587

5 Punia RS, Kundu R, Dalal U, Handa U, Mohan H. Pulmonary hydatidosis in a tertiary care hospital. Lung India 2015;32(03): 246-249

6 Zaman K, Mewara A, Kumar S, et al. Seroprevalence of human cystic echinococcosis from North India (2004-2015). Trop Parasitol 2017;7(02):103-106

7 Rao SS, Mehra B, Narang R. The spectrum of hydatid disease in rural central India: an 11-year experience. Ann Trop Med Public Health 2012;5:225-230

8 Haliloglu M, Saatci I, Akhan O, Ozmen MN, Besim A. Spectrum of imaging findings in pediatric hydatid disease. AJR Am J Roentgenol 1997;169(06):1627-1631

9 Morar R, Feldman C. Pulmonary echinococcosis. Eur Respir J 2003; 21(06):1069-1077 